\title{
Formación universitaria intercultural para indígenas mayas de Yucatán, México
}

\section{Francisco J. Rosado-May}

Universidad Intercultural Maya de Quintana Roo

francisco.rosadomay@uimqroo.edu.mx

RESUMEN

Mediante una descripción vivencial de la construcción de la Universidad Intercultural Maya de Quintana Roo, este trabajo presenta importantes decisiones tomadas y caminos andados para la implementación de un modelo educativo, intercultural, en un contexto de alto rezago y pobreza de los indígenas mayas en Quintana Roo. Sin descuidar las normas y políticas que aplican a instituciones públicas en México, los esquemas de financiamiento, arreglo institucional y pedagogía intercultural, desarrollados en la UIMQRoo, tomaron en cuenta elementos de aprendizaje/transmisión y de construcción/ innovación de conocimiento que combinan aspectos de la cultura local con la educación convencional. Altos indicadores de retención, titulación, empleabilidad y calidad de los programas educativos, así como opiniones de egresados, alcanzados de febrero 2007 a febrero 2015, hacen pensar que las decisiones condujeron hacia la dirección correcta.

Palabras clave: educación superior intercultural, indigenas mayas, educación indígena, pedagogía intercultural. 


\title{
Intercultural higher education for indigenous Yucatec Maya in Mexico
}

\author{
SUMMARY
}

This work presents important decisions made during the implementation of the intercultural educational model at the Universidad Intercultural Maya de Quintana Roo, Mexico, in a context of poverty and conditions of lagging behind the rest of the society of the indigenous Yucatec Maya population. Within the legal framework of public universities in Mexico, the financing of projects, the developing of intercultural pedagogy and designing the institutional settings, rested on elements that included learning/transmission and construction/innovation of knowledge that combined aspects of the local culture with conventional education. High figures on indicators such as retention, graduation rate, employability, and quality of the academic programs, along with opinions from alumni, achieved during the period of February 2007 to February 2015, indicate that the decisions and actions taken in the beginning of the construction of the intercultural model at UIMQRoo, were in the right direction.

Keywords: intercultural higher education, indigenous Maya, indigenous education, intercultural pedagogy. 


\section{INTRODUCCIÓN}

Con el fin de disminuir el rezago en que se encuentra la población indígena del estado de Quintana Roo, México, la Universidad Intercultural Maya de Quintana Roo (UIMQRoo) fue creada el 30 de octubre de 2006. La idea era que, a través de educación con un modelo intercultural, los aspectos estructurales que previenen la adecuada participación e inclusión de las comunidades indígenas para alcanzar un desarrollo sostenible pudiesen ser entendidos y atendidos con mayor probabilidad de éxito.

Para alcanzar el objetivo de la creación de la UIMQRoo, fue muy importante establecer las bases de su funcionamiento y la implementación de su modelo educativo intercultural, por lo que las decisiones y acciones en sus primeros años de operación son considerados altamente críticos en el proceso de creación y consolidación de la UIMQRoo. A través de un ejercicio basado en una descripción autobiográfica, este trabajo presenta algunos resultados que reflejan acciones y decisiones que se llevaron a cabo en los primeros ocho años de vida de la universidad, de febrero de 2007 a febrero de 2015. El autor, rector fundador, también presenta reflexiones y análisis del proceso que permiten conocer el contexto de la creación y operación de la universidad con la expectativa de que las experiencias contribuyan al diseño de nuevas acciones y decisiones de modo que la UIMQRoo alcance con mayor pertinencia, pertenencia, calidad y calidez, el objetivo por el cual fue creada.

Aun cuando el decreto de creación de la UIMQRoo se publicó el 30 de octubre de 2006, no fue sino hasta mediados de agosto de 2007 que iniciaron las actividades académicas con 230 estudiantes de origen maya, distribuidos en tres programas educativos: Lengua y Cultura, Turismo Alternativo, y Agroecología (Rosado-May, 2017). La UIMQRoo se enmarca en el proceso de creación de las universidades interculturales (UI), cuya historia se remite al año 1992, cuando 
los legisladores mexicanos reformaron el artículo 4 constitucional, reconociendo que «México es una nación pluricultural sustentada originalmente en sus pueblos indígenas. La ley protegerá y promoverá el desarrollo de sus lenguas, culturas, usos, costumbres, recursos y formas específicas de organización social». En el año 2001, el artículo 2 constitucional también se reforma señalando, entre otros elementos, que:

La Federación, los Estados y los Municipios, para promover la igualdad de oportunidades de los indígenas y eliminar cualquier práctica discriminatoria, establecerán las instituciones y determinarán las políticas necesarias para garantizar la vigencia de los derechos de los indígenas y el desarrollo integral de sus pueblos y comunidades, las cuales deberán ser diseñadas y operadas conjuntamente con ellos. Para abatir las carencias y rezagos que afectan a los pueblos y comunidades indígenas, dichas autoridades tienen la obligación de: [...] II. Garantizar e incrementar los niveles de escolaridad, favoreciendo la educación bilingüe e intercultural, la alfabetización, la conclusión de la educación básica, la capacidad productiva y la educación media superior y superior. Establecer un programa de becas para los estudiantes indígenas de todos los niveles. Definir y desarrollar programas educativos de contenido regional que reconozcan la herencia cultural de sus pueblos, de acuerdo con las leyes de la materia y en consulta con las comunidades indígenas. Impulsar el respeto y conocimiento de las diversas culturas existentes en la Nación (Casillas Muñoz y Santini Villar, 2006, pp. 81-87).

Atendiendo al reto de atención a poblaciones indígenas, a partir de estas reformas constitucionales, el gobierno mexicano modificó su estructura funcional, cambiando el Instituto Nacional Indigenista (INI) por la Comisión Nacional para el Desarrollo de los Pueblos Indígenas (CDI), y creando el Instituto Nacional de Lenguas Indígenas (Inali) y la Coordinación General de Educación Intercultural y Bilingüe (CGEIB) en la Secretaría de Educación Pública (SEP) (Morales y Gómez, 2009). Estas reformas abrieron el camino para acciones que buscaban generar profundos cambios estructurales que entendiendo y atendiendo los enormes rezagos en los que se encontraba la población indígena en el país. Una de las acciones fue la creación de las UI, que actualmente están presentes, como instituciones públicas, en los estados de Chiapas, Guerrero, Hidalgo, México, Michoacán, Puebla, Quintana Roo, San Luis Potosí, Sinaloa, Tabasco y Veracruz, atendiendo en el ciclo 2015-2016 a 14008 estudiantes (CGEIB, 2017). Esta acción reconoce el importante papel de la educación en el desarrollo del país y abre la posibilidad de crear un modelo educativo diferente, más cercano a la lengua, la cultura y los procesos cognitivos de las comunidades indígenas. La 
creación de las UI en México fue, en general, bien recibida por las comunidades donde fueron construidas, tal y como se desprende a las aportaciones que los rectores fundadores de las universidades interculturales de los estados de México, Chiapas, Puebla, Quintana Roo, San Luis Potosí, Hidalgo, y el primer secretario académico de la UI de Tabasco, y del tercer rector de la UI de Guerrero hacen en la obra de González González et al. (2017).

Reconociendo que en México una universidad representa una derrama económica ${ }^{1}$ (limitada pero importante) en las comunidades donde se asienta, este trabajo asume que la educación juega un papel relevante en el desarrollo de un país, pero asume también que el modelo educativo es determinante para lograr el tipo de desarrollo que se busca, adecuado para una región o para una comunidad. La premisa es que el modelo educativo intercultural puede tener la flexibilidad para entender y atender los grandes rezagos estructurales, funcionales y cognitivos que pueden y deben ser abordados para facilitar e impulsar un mayor y mejor desarrollo sostenible en las comunidades indígenas. Como ejemplo se presenta el caso de la UIMQRoo, donde se llevaron a cabo acciones que, apegadas a la normatividad que aplica a las IES en México, se implementaron decisiones que se reflejan en la estructura de funcionamiento articulados a pedagogías interculturales que guiaron la formación de sus estudiantes. Asimismo, este trabajo enlaza resultados de la gestión rectoral, en la creación y consolidación de la universidad, con conceptos importantes como el de acción afirmativa, pobreza, paternalismo, enfoque intercultural y su relación con procesos cognitivos presentes en comunidades indígenas.

\section{POBLACIÓN MAYA EN QUINTANA ROO}

Usando diversos criterios para determinar la población indígena en Quintana Roo, para el año 2000 el resultado del censo del Inegi señala que el 39,11\% de la población en el estado es indígena. En 2015, bajo el criterio de autoadscripción, en Quintana Roo el 44,4\% de la población total (1 503 009) se considera indígena, de los cuales el 86,7\% es maya yucateco (Inegi, 2015). La población de México en 2015 fue de 119530753 habitantes, de los cuales el 21,5\% se autorreconoce como indígena (Inegi, 2015).

\footnotetext{
No solo la derrama económica es por la inversión en infraestructura y creación de empleos directos en la universidad, sino que también en la comunidad se incrementa la oferta de renta de habitaciones, venta de alimentos y número de negocios, ofreciendo servicios de internet, entre otros efectos en la economía local.
} 
El otro contexto de la población indígena es de pobreza y rezago en varios rubros. Con base en diversos indicadores, la Comisión para el Desarrollo de los Pueblos Indígenas (CDI) en México publica regularmente el Índice de Desarrollo Humano de los Pueblos Indígenas (IDHPI); la base de datos del año 2000 señala para Quintana Roo un valor medio de 0,7398 para los municipios de más alta población indígena (Carrillo Puerto, Morelos y Lázaro Cárdenas). Este valor en el país es de 0,8144 (CDI, 2017). Schmelkes (2013) reporta un valor de 0,7057 para el IDHPI, mientras que para la población no indígena el mismo índice tiene un valor de 0,8304 , es decir, una diferencia de $13 \%$. La misma tendencia persiste al comparar los valores concretos de educación, salud, ingresos y empleo. Estos valores son muy bajos e insuficientes para una vida digna, de acuerdo con Schmelkes (2013) citando a PNUD (2010). Por otra parte, el Consejo Nacional para la Evaluación de la Política de Desarrollo Social publica una base de datos que muestra la medición de la pobreza en México entre los años 2010 y $2014^{2}$. Los datos señalan que en 2010 se registró un $11,3 \%$ de población en extrema pobreza y un $46,1 \%$ en pobreza en el país; para 2014 se registró una reducción en extrema pobreza, alcanzando un valor de 9,5\%, mientras que la población en pobreza aumentó a 46,2\%. En 2010 se registró en Quintana Roo un 34,6\% de población en extrema pobreza y un $6,4 \%$ en pobreza; para 2014 aumentó la extrema pobreza, alcanzando al 35,9\% de la población, y también se incrementa la pobreza, que sube a 7,0\%. Es decir, la pobreza no solo persiste a través de varios gobiernos, sino que se incrementa.

Además de situaciones de pobreza y bajo índice de desarrollo humano, la población indígena mexicana está sujeta a presiones de corte psicológico, fortalecidas por un ambiente de racismo, discriminación y falta de respeto a los derechos humanos (Carrillo Trueba, 2009; Castellanos Guerrero, 2000). Lamentablemente, de acuerdo con Velazco Cruz y Baronnet (2016), ciertas escuelas reproducen esos esquemas, ya sea por contenidos curriculares o por prácticas cotidianas en las interacciones entre grupos sociales. La presión psicológica de denostación, de mensajes constantes de inferioridad racial, lingüística, cultural, color de piel e incluso cognitiva, por siglos, explica una conducta de condicionamiento que fortalece el papel de los no indígenas, también asumidos como los «blancos» (Balcázar et al., 2011). Es importante hacer visible esta situación de presión que tienen los indígenas porque las UI, incluyendo a profesores, administrativos y estudiantes, no están exentas de estos prejuicios.

http://www.coneval.org.mx/Medicion/MP/Paginas/AE_pobreza_2014.aspx (consultado el 12 de marzo de 2017). 


\section{CAMBIOS ESTRUCTURALES PARA ATENDER REZAGOS EN EL DESARROLLO DE COMUNIDADES INDÍGENAS}

La reforma al artículo 2 Constitucional en México, como se mencionó anteriormente, trajo consigo cambios en la estructura funcional del gobierno (e.g. la creción de la CGEIB, CDI e Inali). Si bien esos cambios visibles en el organigrama de funcionamiento del Estado son importantes, no necesariamente atienden los aspectos estructurales no visibles de la exclusión que caracteriza a las comunidades indígenas en el desarrollo del país en general. Con base en los estudios de López y Scott (2000) y Abercrombie et al. (2014, pp. 326-327), se puede asumir que las características de pobreza y bajo índice de desarrollo humano que tiene la población indígena en México pueden considerarse como el resultado que emerge de un arreglo social, en el que están reflejados procesos cognitivos y valores culturales, sociales, económicos, e incluso políticos.

Este arreglo social conforma una estructura que se mantiene y retroalimenta con las acciones de los individuos que la integran. Bajo esta consideración, los cambios en la estructura funcional de un gobierno no necesariamente pueden por sí mismos modificar el resultado que emerge del arreglo social. Es decir, además del cambio estructural funcional también se requieren cambios conceptuales y operacionales, especialmente dirigidos a modificar los paradigmas cognitivos dominantes, y la forma de aprendizaje, construcción, innovación y transmisión de conocimiento. Es en este rubro donde las UI pueden - y deben - jugar un papel preponderante.

Así, mejorar las condiciones sociales de los pueblos indígenas implica reconocer, re-encontrarse, re-valorar, re-aprender y re-adaptar procesos cognitivos que conduzcan a la generación de conocimiento, basado en la interacción de la cultura originaria con el método científico. Este conocimiento puede tener el valor de crear puentes interculturales para un mejor entendimiento social y crear condiciones para el desarrollo sostenible. Es decir, no se trata únicamente de que más jóvenes indígenas tengan acceso a educación sino de crear condiciones para el desarrollo de un proceso cognitivo apropiado culturalmente para el aprendizaje, creación, innovación y transmisión de conocimiento. El proceso de construcción cognitiva intercultural, como una forma para dar nombre al proceso que emerge de la interacción de la cultura originaria de construcción de conocimiento con el método científico, ofrecería bases sólidas para guiar la estructura funcional de las instituciones educativas, la pedagogía, así como la calidad, pertinencia y pertenencia del conocimiento generado. También ofrecería bases sólidas para que 
los tomadores de decisión, con respecto al presupuesto destinado a educación intercultural, al perfil y capacitación del personal administrativo, académico y al nombramiento de directivos, no descansen en lo político sino en la misión que la Constitución otorga a las UI. Es sobre estas comprensiones que se construyó la propuesta de la UIMQRoo.

Cabe señalar que diversas organizaciones internacionales, como el Fondo Internacional de Desarrollo Agrícola (IFAD, 2003), el Foro Económico Mundial (WEF, 2017), el Banco Interamericano de Desarrollo (BID, 2012) y otros están dirigiendo la mirada a los sistemas indígenas de conocimiento, no solo para que los indígenas participen en el desarrollo global sino para que aporten conocimiento y experiencia desde sus saberes y formas de saber. Sobrevila (2008) señala que alrededor del $80 \%$ de la biodiversidad de nuestro planeta se encuentra en alrededor del $20 \%$ de territorio que está en las manos y decisiones de pueblos originarios. Toledo (2013) confirma lo anterior y señala que la explicación radica en las formas de construcción de conocimiento que tienen los pueblos originarios. La biodiversidad juega un papel importante en la mitigación de los efectos del calentamiento global y en la producción de alimentos; la diversidad biológica no se explica sin la diversidad cultural que aportan los pueblos indígenas. Por estas y otras razones, Shaw et al. (2009) reconocen el papel del conocimiento indígena en la reducción significativa de riesgos de desastres naturales.

Con base en el análisis anterior, es evidente que no basta solo con ofrecer espacios educativos para incrementar el número de estudiantes indígenas en las universidades interculturales e inclusión física en educación superior, sino que también debe haber inclusión de sus saberes y formas de construcción de sus sistemas de conocimiento. Es decir, no basta con asumir que estructuralmente se están atendiendo rezagos de la población indígena a través de educación superior, si el concepto de estructura solo involucra lo funcional y no el proceso cognitivo de construcción de conocimiento, lo invisible. Y no hay mejor alternativa que el modelo educativo intercultural para hacer posible la transformación estructural que requiere la población indígena.

\section{CONTEXTO DE POLÍTICA EDUCATIVA EN LA IMPLEMENTACIÓN DE} LAS UI

La UIMQRoo se ubica en la categoría de universidad pública estatal de apoyo solidario (UPEAS), la cual es interpretada por la federación como una acción de solidaridad federal a una iniciativa estatal, desde el punto de vista financiero 
(Rubio Oca, 2006). Para el funcionamiento de la institución, en teoría, la federación aporta una cantidad semejante a la que aporte el estado, pero en la práctica, a veces el estado aporta más, o a veces la federación aporta más de lo que pone la otra parte. El modelo fue creado en 1978 como una forma de incrementar la eficacia institucional motivando la planeación estratégica y también inhibir la corrupción y opacidad en las instituciones de educación superior (IES) en México (Mejía Pérez, 2016) Entre los años 2001-2012, cuando en materia de educación debió reflejarse el cambio estructural a raíz de la reforma de los artículos 2 y 4 constitucionales, la política oficial puso énfasis en el crecimiento de la matrícula, impulsando especialmente el modelo educativo tecnológico (Mendoza-Rojas, 2015). No hay evidencias de esfuerzos gubernamentales claros y eficaces en materia de cambios estructurales que conduzcan a mejorar la calidad o a entender los procesos cognitivos de los pueblos originarios ni sus formas de construcción, innovación y transmisión de conocimiento. Lo anterior no se explica por la falta de información acerca de formas de aprendizaje y construcción de conocimiento en comunidades indígenas; Mallea y Young (1984) y Rogoff (2003) habían publicado libros clásicos que presentaban información científica sobre este tema. Otros ejemplos son estudios sobre el aprendizaje entre los mazahuas y otros grupos indígenas identifican diferentes estrategias de aprendizaje entre los niños, que se aplican de una forma dinámica y múltiple en tiempo y espacio (Paradise, 1987; Paradise y De Haan, 2009).

Entre las estrategias están las habilidades de observación, la incorporación temprana en actividades que requieren responsabilidad, la práctica como base del aprendizaje, la creación de un ambiente seguro en el que echar a perder es parte del aprendizaje y permitir que cada niño aprenda según su propio ritmo (Paradise, 1994, publicado antes de la creación de las UI; Paradise y De Haan, 2009, publicado poco después de la creación de la UIMQRoo). Además, las publicaciones de varios autores antes de la apertura de las UI, entre los que destacan Gaskins (1999) Chavajay y Rogoff (2002), Rogoff (1981) y Rogoff (1990), al estudiar los mayas de Yucatán y otros grupos indígenas, encuentran elementos de aprendizaje semejantes a los expuestos anteriormente. Prácticamente los mismos autores reportan investigaciones años después que confirman sus primeros reportes (Gaskins, 2003; Gaskins y Paradise, 2010; Rogoff, et al., 2014), por lo que antes y después de la creación de las UI ya había información disponible sobre procesos de aprendizaje y construcción de conocimiento en comunidades indígenas.

Treinta y nueve años después de la creación de las UPEAS se crean las UI sobre la misma base administrativa, sumándose al cada vez más elevado número 
de UPEAS en el país que están entre el aislamiento y la precariedad (Mejía Pérez, 2016). El acceso a financiamiento es uno de los temas más importantes que abordan los rectores fundadores de varias UI de México en la obra de González González et al. (2017), considerado como uno de los factores limitantes del desarrollo del modelo intercultural. Cada año los rectores recorren instancias estatales y federales, tanto del Poder Ejecutivo como del Legislativo, incluyendo la Cámara de Diputados y de Senadores, para asegurar un financiamiento básico, que no siempre se logra incrementar o incluso mantener. Cada año también se hace el planteamiento para establecer una política de presupuesto multianual para la educación, de forma tal que otorgue certidumbre para la planeación y logro de objetivos.

No solo el acceso a financiamiento adecuado es una situación que enfrentan las UI, sino también está la competencia con otros sistemas y modelos educativos que el gobierno ha fomentado en regiones indígenas. Tomando como base los campus y sedes principales de las instituciones de corte tecnológico con las los campus y sedes principales de las UI, existen 416 opciones en el país que presentan el sistema de educación superior tecnológica, en comparación con las 36 de las UI. Esta situación, de acuerdo con Rosado-May (2015a), se puede interpretar como un indicador de que la visión del gobierno no favorece el desarrollo del modelo intercultural. Muchas opciones de educación tecnológica están presentes en regiones con alta población indígena; Quintana Roo es un ejemplo, existen trece opciones de educación tecnológica pública y solamente una intercultural, incluso con distancias muy cercanas a lugares donde está asentada la UIMQRoo y con oferta educativa similar.

Al analizar el contexto que tienen los académicos de las UI con respecto a los de otros sistemas educativos, se encuentra lo siguiente. Los académicos de las UI deben sujetarse a los mismos procedimientos de evaluación de su desempeño y recibir reconocimientos y apoyos extraordinarios semejantes que los académicos de otros sistemas educativos. Por ejemplo, en México destacan el Programa para el Desarrollo Profesional Docente para el Tipo Superior (Prodep), bajo el auspicio de la Secretaría de Educación Pública (SEP) y el Sistema Nacional de Investigadores, bajo el auspicio del Consejo Nacional de Ciencia y Tecnología. El Prodep tuvo como antecesor el Programa de Mejoramiento del Profesorado (Promep). Las evaluaciones hechas por la SEP (2006), por Moreno et al. (2016) y por Zogaib Achcar (2000) permiten afirmar que, en términos generales, esos programas permitieron incrementar la habilitación académica de los profesores universitarios en México y facilitaron el proceso de planeación institucional, especialmente en materia de investigación y producción académica. 
No obstante, los criterios usados se basan, por un lado, en el modelo cognitivo dominante, convencional; no toman en cuenta los procesos de los pueblos originarios. Por otro lado, se usan indicadores de desempeño establecidos para instituciones consolidadas y no para aquellas que apenas inician. Además de estas desventajas en materia de evaluación para los académicos de las UI, es importante notar que el componente de Promep para el fortalecimiento de la habilitación académica de los profesores universitarios no ha aplicado a las UI; es decir, no ha habido apoyos para que profesores de las UI alcancen su maestría o su doctorado, dependiendo del grado académico que posean al ingresar a la UI. ¿Cómo, entonces, lograr una educación pertinente y de calidad? Otro caso es el de un incentivo que reciben profesores de las universidades públicas estatales conocido como el Programa de Estímulos al Desempeño del Personal Docente; este programa no se ha aplicado al personal académico de las UI.

Por otra parte, prácticamente el $100 \%$ de los profesores-investigadores de las UI, al menos hasta el año 2015, se formaron con base en el proceso cognitivo convencional $^{3}$ del método científico. Ante este escenario, sería lógico pensar que el sistema de educación intercultural desarrollaría un programa de capacitación para esos académicos, incluso desde antes de la creación de la primera UI en 2004, e impulsaría publicaciones dando a conocer la evolución del proceso de formación, los resultados, las prácticas pedagógicas eficaces y las no eficaces, etc. De la poca investigación disponible sobre la capacitación para la interculturalidad, Bertely Busquets (2011) revisa el estado del arte de la educación superior intercultural en México, señalando que, si bien hay una formación para profesores indígenas y para nivel educativo básico, no la hay para nivel superior, y detecta desequilibrios y conflictos entre el enfoque intercultural oficial y las opciones y programas universitarios de las UI. La formación intercultural debe estar articulada con los mecanismos de evaluación para promoción e incentivos (Caballero Rodríguez, 2013). Adicionalmente, la formación intercultural del personal administrativo es casi nula. Si bien es cierto que el personal administrativo tiene tareas marcadas por procedimientos y legislación estatal y federal, y que no reconoce diversidad cultural, también es cierto que en la cotidianidad de sus actividades requieren entender los procesos interculturales que impulsa la institución y también conocer los procesos cognitivos de los académicos y de los estudiantes.

Se adopta la expresión de Mato (2012) para entender que el modelo convencional es aquel que no incluye en sus planes de estudio, o en sus pedagogías, los conocimientos, saberes, idioma, cultura o la cosmovisión de los pueblos indígenas. 


\section{LA UIMQROO 2007-2015: CIMIENTOS DEL DISEÑO INSTITUCIONAL PARA EL CAMBIO ESTRUCTURAL EN COMUNIDADES INDÍGENAS}

Entre los años 2007 y 2014, cada año el Consejo Directivo, máximo órgano de gobierno de la universidad, integrado por representantes del gobierno federal, estatal, municipal y sociedad civil, incluyendo representantes de comunidades indígenas y locales (UIMQRoo 2010), recibía un informe que incluía indicadores de desempeño institucional. En diciembre de 2014, algunos de los logros alcanzados por la UIMQRoo con respecto a indicadores establecidos por la SEP, y señalados en el informe anual, fueron el $100 \%$ de titulación de egresados; alrededor de $70 \%$ de eficiencia terminal en la misma cohorte; $100 \%$ de programas educativos evaluables que alcanzaron nivel I de los Comités Interinstitucionales de Evaluación de la Educación Superior $\left(\right.$ CIEES $\left.^{4}\right)$; cerca de $80 \%$ de profesores con reconocimiento del programa Prodep; alrededor de $85 \%$ de estudiantes de las primeras tres promociones que fueron los primeros en su familias en alcanzar un título universitario; alrededor de 150 comunidades de donde provienen los estudiantes de la UIMQRoo y donde se llevan a cabo actividades de investigación y gestión, y alrededor del $90 \%$ de empleabilidad de los graduados. Además, con relación al recurso financiero para la operación de la institución, el modelo permitió alcanzar un valor cercano al 50\% del total proveniente de proyectos que incluyeron recursos extraordinarios concursables y fondos de organismos internacionales como a Fundación Kellogg.

El incremento de la autoestima de los estudiantes los conduce a alcanzar metas más allá de una licenciatura. Un ejemplo de lo anterior se encuentra en los datos de participación de egresadas de la UIMQRoo en el Programa de Fortalecimiento Académico para Indígenas a través de la Incorporación de Mujeres Indígenas al Posgrado Nacional para el Fortalecimiento Regional, proyecto federal auspiciado por el Consejo Nacional de Ciencia y Tecnología ${ }^{5}$, que se ha implementado anualmente desde 2014, en Quintana Roo. En 2014, de las veinte jóvenes seleccionadas, catorce fueron egresadas de la UIMQRoo; en 2015, de

4 Los CIEES se integran por pares académicos con alto reconocimiento y con perfil adecuado para evaluar la calidad de los programas educativos, también conocidos como carreras. Existe una metodología consensada entre las instituciones de educación superior, que toma en cuenta estándares internacionales, para definir el nivel de calidad que se otorgaría al programa evaluado. El nivel más alto es el I, lo que indica que el programa puede optar al siguiente nivel que es el de acreditación.

5 http://www.coqcyt.gob.mx/portal/WebPage.php?Variables=Pagina-Nota|IdNota-5858 (consultado el 30 de septiembre de 2017). 
las veinticinco jóvenes seleccionadas el 56\% fueron de la UIMQRoo; en 2016, ninguna de las jóvenes seleccionadas fue de la UIMQRoo.

Conocer a los estudiantes, su entorno, su cosmovisión, así como su sistema de aprendizaje y construcción de conocimiento, fueron elementos clave para alcanzar los indicadores antes mencionados, pero no los únicos. Fue muy importante encontrar la forma en la que esos elementos podrían articularse con las expectativas, lineamientos y políticas educativas de la SEP y del gobierno del estado, y plasmarlo de la mejor forma posible en el arreglo institucional y andamiaje normativo de la UIMQRoo.

Sabiendo de las limitantes por el tipo de financiamiento que recibe la UIMQRoo, la estrategia para alcanzar cerca del $50 \%$ del presupuesto ejercido, proveniente de fuentes competitivas gubernamentales y no gubernamentales, fue contar con académicos de alto nivel, capacitados para buscar y atraer recursos, programar al menos dos días enteros de la semana para actividades de investigación o vinculación de modo que los compromisos de los proyectos se puedan cumplir, propiciar trabajo inter y multidisciplinario con la participación de estudiantes y preferentemente en sus comunidades, impulsar propuestas de proyectos basados en principios de interculturalidad para crear un nicho conceptual y metodológico que no podían ocupar otras IES, así como el trabajo transparente y con rendición oportuna de cuentas. La normatividad permitió la implementación eficaz de la estrategia, de modo que el equipamiento de laboratorios, investigación, parque vehicular, participación en reuniones académicas nacionales e internacionales, intercambio académico de profesores y estudiantes, incluso viajes de estudio, fueron actividades financiadas por esta estrategia.

El ingreso de los académicos y administrativos fue mediante convocatorias públicas y concurso de oposición, con la evaluación de un comité integrado por académicos o administrativos según el caso, de la UIMQRoo, de otras IES, estudiantes y, algo innovador, representantes de las comunidades locales. Los académicos ingresaron así a un sistema de permanencia mediante evaluación que no solo medía parámetros oficiales como publicaciones y evaluaciones de los estudiantes, sino que incluía sus contribuciones al proceso de construcción de interculturalidad y su impacto en las comunidades del entorno universitario. Los estudiantes ingresaron tomando la evaluación oficial conocida como EXANI II y una evaluación interna, llamada Evaluación Institucional de Ingreso a la Universidad (EVIIU), diseñada por psicólogos de la UIMQRoo que se entrenaron en el Centro Nacional de Evaluación para la Educación Superior A.C. Este instrumento busca medir las «ganas», como se dice coloquialmente en la universidad, 
el deseo de superación de los futuros estudiantes de UIMQRoo. Al momento de decidir quienes ingresan a la UIMQRoo, el comité de selección otorga más peso al EVIIU que al EXANI II.

El arreglo institucional contempla como estructura funcional la mayor horizontalidad posible: la figura del rector, cuatro direcciones (Académica; Planeación y Desarrollo Institucional; Jurídica, y Administración y Servicios), y cinco departamentos académicos (Lengua y Cultura; Vinculación; Desarrollo Sostenible; Centro Intercultural de Proyectos y Negocios, y Salud y Desarrollo Comunitario). Los profesores se adscriben a uno de los departamentos que a su vez hospeda cuerpos académicos. Existe un Consejo Departamental y una coordinación por cada uno de los programas educativos, que coadyuvan en la planeación y toma de decisiones académicas.

Uno de los principales elementos de la organización institucional fue la decisión de cuáles y cuántos programas educativos podría desarrollar la universidad. El modelo educativo intercultural propuesto por el gobierno consta de cuatro programas educativos: Lengua y Cultura, Desarrollo Sustentable, Comunicación Intercultural y Turismo Alternativo (Casillas Muñoz y Santini Villar, 2006, p. 188202). La UIMQRoo inició en 2007 con Lengua y Cultura, Sistemas de Producción Agroecológicos, y Turismo Alternativo. En 2009 se ofreció, adicionalmente, Salud Comunitaria y Gestión Municipal. En 2012 se amplió la oferta con los programas: Tecnología de Información y Comunicación; Gestión y Desarrollo de las Artes, y Desarrollo Empresarial, y se abrió la maestría en Educación Intercultural. Ocho licenciaturas y una maestría, abiertas en cinco años, se explican por dos razones fundamentales: la primera: con solo tres o cuatro programas el mercado laboral se satura muy pronto y por tanto impacta negativamente en la matrícula de ingreso; la segunda y más importante: para cumplir con la misión encomendada a las UI se requiere el concurso y la formación de una masa crítica en todas las áreas de conocimiento que cubra los tres sectores de la economía ${ }^{6}$. Así, para la UIMQRoo el desarrollo sostenible, con crecimiento económico de la región, debe emerger de la interacción y articulación de los programas educativos creados, tomando en cuenta los tres sectores de la economía.

La crítica recibida por el «alto» número de programas educativos se basa en que no se deben abrir tantos programas, porque su sostenimiento financiero $\mathrm{y}$

\footnotetext{
6 Sector primario, producción agrícola (e.g. Agroecología); sector secundario, transformación de productos primarios (e.g. Desarrollo Empresarial), y sector terciario: servicios (e.g. Turismo Alternativo).
} 
demanda de estudiantes sería muy difícil de mantener. La respuesta a esta crítica no solo está en la necesidad de desarrollar diferentes áreas de conocimiento para que las UI y sus egresados puedan realmente contribuir al desarrollo de la región; también está en el propio modelo educativo. La idea fue tener programas educativos y perfil de profesores, con base en área de conocimiento, no por especialidad. De esta forma, al identificar que un programa educativo ha agotado su pertinencia, se podría poner en pausa para, básicamente con los mismos profesores, la misma infraestructura y algunos cambios en el plan de estudios, convertirse en uno nuevo y con mayor pertinencia. Por ejemplo, el programa de Gestión Municipal, diseñado para fortalecer las capacidades y masa crítica en la gobernanza municipal, podría saturar el mercado laboral en pocos años. La alternativa es convertir el programa a otro dentro de la misma área de conocimiento, como por ejemplo Derecho con Enfoque Intercultural. Es importante mencionar que las alternativas a esta propuesta, sea para evitar el crecimiento en número de programas educativos o para crecer sin cerrar programas, no son ni viables, ni adecuadas, ni pertinentes.

El diseño del plan de estudios de cada programa educativo a nivel de licenciatura básicamente se integró por tres etapas, con un total de cuatro años. La primera, de un año, conocida como Formación Básica, tiene el objetivo no solo de fortalecer los conocimientos y habilidades de los estudiantes para su formación superior, sino que, muy importante, se trató de lograr una cohesión entre estudiantes y la recuperación de su autoestima como indígenas, así como reconocer y desplegar su potencial cognitivo. Esta motivación explica, en gran medida, los exitosos indicadores que alcanzó la UIMQRoo: la reducción de este año para favorecer la especialización temprana sería altamente negativa para los estudiantes y para las tendencias de perfil que demanda el mercado laboral actual y futuro. La segunda etapa, un año y medio adicional, se dirige a la formación de nivel de profesional asociado ${ }^{7}$. La tercera etapa, otro año y medio, fue diseñada para la formación de licenciatura. Los estudiantes cursaban dos ciclos de dieciséis semanas por año y un curso-taller de vinculación comunitaria durante el verano, con trabajo de tiempo completo durante ocho semanas en una comunidad.

Desde el punto de vista de apoyo pedagógico, la normatividad interna de la UIMQRoo contempló la contratación de profesores — conocidos como sabios

7 En México se usa el término profesional asociado para el sistema universitario, y el término técnico superior para el sistema tecnológico. Ambos se refieren al nivel internacionalmente conocido como 5B, mientras que la licenciatura es 5A. El profesional asociado, o técnico superior, es un nivel académico previo a la licenciatura y puede recibir una cédula profesional. 
locales - sin formación académica formal, quienes co-enseñan co-laborando con profesores con grados de maestría o doctorado. Asimismo, el sistema contempla la participación de abuelos tutores (en maya, nool iknal), quienes son elegidos por los estudiantes, normalmente personas de mucho respeto y conocimiento de su propia comunidad, quienes dan seguimiento a la formación de los estudiantes (Rosado-May, 2012). Con sabios locales como profesores y el $\mathrm{iknal}$, se establecieron condiciones para fomentar el entrenamiento en la observación y la práctica como aspectos fundamentales en el proceso de aprendizaje y construcción de conocimiento que han desarrollado los mayas. Los profesores entendieron las formas cognitivas preferenciales de los estudiantes y sobre esa base diseñaron actividades de construcción de conocimiento. En este sentido, los profesores pudieron detectar cuándo los estudiantes prefieren aprender y crear conocimiento a través de la inducción (partiendo de la práctica) y cuándo a través de la deducción (partiendo de la teoría). Los profesores se capacitaron para detectar cuando un estudiante cambia, dependiendo del tema, de una forma preferencial de aprendizaje a otra. La idea es facilitar el proceso de aprendizaje y construcción de conocimiento tomando como base los procesos cognitivos de los estudiantes.

\section{VOCES DE EGRESADOS, PRIMEROS RESULTADOS DEL MODELO INTERCULTURAL}

Se presentan cuatro ejemplos que ilustran el espíritu, expectativas y resultados que han tenido estudiantes y egresados de la UIMQRoo: dos hombres y dos mujeres, cada uno de diferente programa educativo. Tres de ellos tuvieron el maya como primera lengua y dos tuvieron experiencia de intercambio académico internacional. Los cuatro son de origen humilde y fueron los primeros en sus familias en alcanzar un título universitario.

NCP es originaria de un asentamiento ubicado a unos $12 \mathrm{~km}$ de Señor, la población más cercana, municipio de Felipe Carrillo Puerto (FCP). Ahí creció hablando exclusivamente maya. A la edad de siete años inició sus estudios de primaria en Señor, donde empezó a hablar español. En bachillerato, su dominio del español aún no era total; no obstante, NCP obtuvo resultados sobresalientes. Cuando NCP supo de la UIMQRoo tuvo muchas dudas, tardó en decidirse. Entre las razones de sus dudas estaban: (i) la inseguridad sobre su capacidad de desempeño en el nivel superior; (ii) el poco dominio del español para un buen nivel académico; (iii) tener que dejar su comunidad y su familia, y (iv) el aspecto de financiamiento para sus estudios. En sus palabras: 
Yo tenía el deseo de estudiar desde pequeña; mis padres me inculcaron mucho ese deseo. Me imaginaba que era difícil, sobre todo porque no hablaba bien el español y se me dificultaba leer y entender. Siempre escuché de mis compañeros y de vecinos que estudiar no era para nosotros los mayas, que solo perderíamos el tiempo y no aprenderíamos gran cosa. Insistían en que es mejor aprender un oficio o casarse y que solo los hombres tenían la capacidad intelectual para estudiar. Cuando supe de la UIMQRoo no me animé al principio, pero escuché muchas opiniones de profesores que respeto mucho, y me animé porque me convencí de que ahí no habría discriminación y que si daba lo mejor de mí tendría la oportunidad de viajar a otros países (algo que nunca imaginé que fuese cierto) $\mathrm{y}$, si se pudiera, de seguir estudiando. Hoy tengo un título universitario, he tenido la oportunidad de viajar a Canadá, Estados Unidos y otras partes de mi país y estado y estoy estudiando mi maestría. Me siento más segura de mi misma, aunque aún percibo discriminación en muchas personas [NCP tiene actualmente veinticuatro años, egresó en 2015 como parte de la quinta generación de licenciados en Lengua y Cultura].

MKM es originaria de la comunidad San Felipe del municipio de José María Morelos (JMM). Ahí vivió hasta los dieciocho años. En su casa sus padres no procuraron enseñarle maya porque pensaban que «aprender español era mejor para salir bien en la escuela». Ella recuerda que decía palabras en maya y su mamá se lo prohibía. Sus padres platicaban en maya para que no los entendiera, lo que hizo que ella se motive por aprender maya. Su familia se cambió a vivir a JMM, lugar donde concluyó el nivel medio superior. Inició la carrera de Administración de Empresas en el Instituto Tecnológico de Chetumal, pero tuvo que abandonarlo porque los recursos económicos no alcanzaban para solventar todos los gastos. Siempre soñó con estudiar una carrera universitaria, pero era prácticamente imposible, era casada, trabajaba y tenía hijos. En esa situación estaba cuando inició actividades la UIMQRoo en JMM. En 2008 MKM decidió inscribirse para cursar una carrera, lo cual hizo con múltiples sacrificios, esfuerzos, superando situaciones familiares y de salud difíciles.

Mis deseos de superarme, de estudiar una carrera, se estaban esfumando y me desesperaba. Recuerdo que desde 2003 se habló mucho en JMM que se iba a crear una universidad aquí, pero como siempre pasa con programas de gobierno, no había seguridad. No podía creer cuando en 2007 se abrió la UIMQRoo, y con una carrera que me podía gustar porque mi padre es campesino. Cuando expresé mi deseo de estudiar fueron muy pocas personas que me apoyaron: la mayoría me dijo que como yo estaba casada, con hijos y trabajando, no era adecuado. También me dijeron que la universidad era para personas inteligentes, que los mayas no podrían con el nivel de estu- 
dio si es que vienen profesores de buen nivel, pero si vienen profesores de bajo nivel seguramente hasta van a regalar los títulos. Sonaba muy fuerte y difícil, pero a la vez muy atractivo. Recuerdo que nos decían que si la universidad fuese «suave» con sus estudiantes entonces no se estaría honrando el potencial de inteligencia que la cultura maya ha demostrado al mundo y que nosotros somos los herederos. A mí me pareció adecuado y dije, lo voy a intentar y lo voy a lograr. Hoy tengo un título universitario, sigo trabajando, aunque no en mi carrera, porque decidí seguir con mi maestría en Educación Intercultural, en la misma UIMQRoo. También veo que mis hijos se sienten orgullosos de su mamá, tengo el apoyo de mis padres y tengo la gran satisfacción de que estoy cumpliendo mi sueño de superación. He estado haciendo investigaciones acerca del proceso de aprendizaje que tienen los estudiantes de Agroecología y he hecho comparaciones entre mayas de Yucatán y de Chiapas, eso nos puede ayudar a diseñar herramientas pedagógicas para la enseñanza de la agroecología en diferentes niveles educativos [MKM tiene actualmente 35 años, egresó en 2013 como parte de la tercera generación de la Ingeniería en Sistemas de Producción Agroecológicos].

MPC es oriundo de Tihosuco, muy cercano a Tepich, sitio donde inició la Guerra de Castas en Yucatán, en 1847, municipio de FCP. Es el hijo mayor de una familia numerosa. Sus padres procuraron para todos los hijos una educación con valores propios de su cultura, de su lengua, responsabilidad, trabajo, compromiso con su comunidad, compartir cosas y conocimiento, respeto a los mayores, a sus semejantes, a la naturaleza y los animales, aprendieron de ofrendas a los dioses de la milpa, y lo difícil —y a la vez fácil — que es producir alimentos. MPC estudió en Tihosuco hasta su bachillerato; al terminar, se sumó a las filas del Consejo Nacional de Fomento Educativo, donde trabajó por dos años en comunidades mayas alfabetizando a niños, pero tomando muy en cuenta su propia lengua y cultura. En 2007 MPC se inscribió en la UIMQRoo; desde entonces, cada año, durante cuatro años, un hermano o hermana de MPC se inscribió en la UIMQRoo. Todos ellos han concursado por una beca para asistir a un curso de verano en Estados Unidos, financiado por la embajada de ese país, y todos ellos han sido beneficiarios de dicho programa.

No sé por qué todos mis hermanos y yo hemos tenido el impulso de seguir aprendiendo, de estudiar, de conocer. Creo que mis padres nos lo inculcaron desde pequeños. Todos hablamos bien maya y trabajábamos en las labores de la casa, de la milpa, del cuidado de los animales, escuchando a los jóvenes que salían de la comunidad para trabajar en la Riviera Maya. Yo pensaba, algún día no solo iré a conocer la Riviera Maya, sino que me iré a otro país para aprender y para saber si de verdad la cultura maya es apreciada más 
en otros países que en su propia tierra. De alguna forma sabía que la mejor forma de hacerlo es estudiando una carrera universitaria y seguir adelante. Yo escuchaba a adultos y jóvenes hablando de lo bien que se gana en la Riviera Maya, pero pensaba: ¿será que sin estudios así será para ellos siempre? Tenía mis dudas, aunque tenía muchas ganas de estudiar sabía que no sería fácil, que estaría en desventaja con respecto a estudiantes de las ciudades con más recursos que nosotros. Para mí, estamos en una nueva era, el 14 Baktun Maya. Esta era nos permite no olvidar que la Guerra de Castas sigue vigente, los estudiantes mayas estamos olvidados, no tenemos muchas oportunidades ni se valora nuestra lengua y cultura como debe ser. Pienso que esta nueva forma de guerra de castas no se está peleando con armas o cañones, sino con estudios, lápiz y cuaderno. Ya tengo un hijo, a quien le enseñaré las costumbres, nuestra lengua maya, y lo animaré a conocer el mundo sin que pierda su propia identidad. Me gusta el lema de mi universidad: «Ser en el mundo, ser nosotros». Nosotros los jóvenes somos los portadores de los ideales de Manuel Antonio Ay, Jacinto Pat, Cecilio Chí, Francisco May y muchos otros héroes mayas que lucharon por nuestra gente, por un futuro sin que seamos esclavos [MPC tiene actualmente 31 años, egresó en 2011, de la primera generación de la licenciatura en Turismo Alternativo].

ITM es originario de JMM, sus padres son de origen maya. Tiene siete hermanos. Desde niño su padre lo llevaba para que observe la forma en que trabajaba, ya sea cultivando o comercializando productos diversos. Recibió una educación familiar basada en valores y coraje para salir adelante en la vida, lo cual forjó en él un carácter fuerte para salir adelante. ITM tuvo necesidad de vivir casi independiente de su familia desde muy joven, pero nunca perdió de vista el gusto por estudiar y forjarse un futuro sólido en el área empresarial. Al terminar su bachillerato, se encontró con que la UIMQRoo ya ofrecía una carrera en negocios; no lo dudó y se inscribió.

La verdad no he tenido una vida fácil pero tampoco he sido infeliz. He aprendido a ser independiente, a forjarme un futuro, trabajar fuerte y alcanzar algunas metas que me he impuesto. Agradezco a mis padres que me hayan enseñado las bases de mi identidad, de mi personalidad. Quizá por las carencias en mi familia, quizá por lo que observé cuando acompañé a mi padre en su trabajo de comercio, o quizá por lo que escuché en alguna plática, lo cierto es que desde muy joven tenía yo el interés de desarrollarme como empresario. Terminé mi bachillerato en el año 2012. Ya para entonces todos sabíamos de la UIMQRoo, pero lo que no sabíamos es que se abriría una carrera relacionada con negocios. Platicando con mis amigos bachilleres escuchaba que varios de ellos preferían salir de JMM, pensando que encontrarían mejores oportunidades de estudio, pero no pensaban en los costos que ello implicaba 
para sus familias, ni tomaban en cuenta los otros costos que implica vivir en lugares grandes. Muchos de ellos, lamentablemente, tuvieron que abandonar sus estudios porque no podían costear los gastos. El hecho de estudiar maya y la cultura como parte de nuestra formación me ha permitido desarrollar ideas para negocios. Cada vez que a mi negocio vienen personas de otras partes y conocen lo que hacemos y cómo llegamos hasta este punto, expresan su admiración y nos da ánimos, nos impulsan. Hoy tengo un negocio propio que está creciendo y estoy a unos cuantos meses de obtener un título universitario; tengo la capacidad de emplear hasta a siete personas, de las cuales cinco son estudiantes. Esta es la mejor ayuda que se les puede brindar: empleo [ITM tiene actualmente veinticinco años, egresó en 2016 como parte la primera generación de Ingeniería en Desarrollo Empresarial].

Si bien cada testimonio resalta la llegada a la UIMQRoo, es importante notar la relación entre lo que señalan sobre sus desempeños profesionales posteriores con el modelo educativo intercultural: elevada autoestima, entendimiento de procesos cognitivos entre culturas, fortalecimiento de su identidad y formación sólida para una sociedad multicultural para coexistir y contribuir. Lograr un nivel de autoestima y seguridad como el que exhiben estos egresados no fue fácil. Parte del proceso implicó un trabajo fuerte con los estudiantes para entender y relacionar epistemológicamente términos mayas con conceptos occidentales, así como contar con bases conceptuales que les permitiesen explicar que los apoyos que reciben no son ni productos ni resultado de una política de sobreprotección a los indígenas. En el primer caso, Rosado-May y Osorio Vázquez (2014, pp. 155-157) presentan el término maya $\mathrm{Ka}$ 'anan kax como la expresión de un concepto que se refiere al manejo del ecosistema selva y el concepto de dinámica sin movimiento para reflejar un dicho común en el área: «despacio que tengo prisa, lento pero seguro», interpretado por los no conocedores al pensar que en las comunidades indígenas no hay cambios a través del tiempo. Si no hubiera cambios, no habría la riqueza de conocimiento de su entorno natural ni de lo que nos maravillamos al leer sobre la cultura maya. En el segundo caso fue importante encontrar una defensa contundente al concepto de «discriminación en reversa» que presentaban aquellos que se oponían a la creación de la UIMQRoo y al acceso de varios apoyos que tenían los estudiantes indígenas. El concepto de «acción afirmativa» — usado en el gobierno del presidente de Estados Unidos, John F. Kennedy, a través de la orden ejecutiva 10925 de 1961 - fue el que se usó en los diversos debates, antes de la creación de la UIMQRoo, y durante su funcionamiento.

Al analizar el concepto, Crosby (2004) incluso presenta el análisis jurídico que se hizo en la Suprema Corte de Justicia de Estados Unidos al atender un caso 
relacionado con la aceptación de un estudiante negro en la Universidad de Michigan, en lugar de un estudiante blanco. La corte falló en favor de la universidad, argumentando que el estudiante negro representaba a un grupo social que aún no tenía el suficiente número de personas que permitiera tener la masa crítica necesaria para que ese grupo social tenga las condiciones de equidad necesarias para desarrollarse mejor en el país. Acción afirmativa es mucho más que la simple política de cuotas: también permite que se ejerza el concepto de justicia cognitiva que muchas veces es ignorado por el sistema educativo. Los pueblos indígenas tienen formas diferentes de aprendizaje y construcción de conocimiento (Rogoff, 2014); por tanto, si en la escuela ese sistema de aprendizaje y construcción de conocimiento no es reconocido, ni apreciado ni potencializado, entonces no hay justicia cognitiva (Aguiló Bonet, 2009).

Acción afirmativa y justicia cognitiva son conceptos que respaldan la creación de las UI en México y se suman a los acuerdos internacionales que ha suscrito nuestro país, como el Acuerdo 169 de la OIT. Con estas bases, México se ve obligado a procurar una educación de calidad para grupos minoritarios como el de los indígenas. Esto quiere decir crear las plazas y ofrecer excelentes salarios para tener los mejores profesores con las más altas credenciales académicas y, al mismo tiempo, exigir resultados en formación de recursos humanos, en investigación y en vinculación.

¿Cuántos años se necesita para formar la masa crítica necesaria entre los mayas u otro grupo indígena? ¿Cuántas generaciones? ¿Qué condiciones? No tenemos respuestas concretas, pero sabemos con certeza que debimos haber empezado hace muchos años y que debemos hacerlo con calidad.

\section{CONCLUSIONES Y RECOMENDACIONES}

El entendimiento de la situación de la población indígena en Quintana Roo, incluyendo aspectos cognitivos, permitió visualizar elementos importantes que fueron atendidos por el modelo intercultural en la UIMQRoo. No hay antecedente de otra IES en el estado que lo haya hecho. Algunas de las decisiones y acciones antes descritas pueden ser consideradas innovadoras, o bien contradictorias, frente a las políticas de educación superior en México. Sin embargo, los indicadores demostraron que los aspectos estructurales, de funcionamiento, cognitivos y pedagógicos se diseñaron en la dirección correcta.

La definición de trabajo adoptada en la UIMQRoo sobre educación intercultural permitió articular los esfuerzos que, en forma integral, atienden los retos y expectativas de la población maya. Rosado-May (2013b, p. 163) considera que 
la UIMQRoo es «un espacio seguro en el que diferentes formas de construcción de conocimiento coexisten con respeto y valores éticos universales, generando oportunidades de sinergias para construir un nuevo conocimiento traducido en conceptos, metodologías, técnicas, tanto para las ciencias como para las diferentes expresiones de artes». Este nuevo conocimiento es intercultural (Rosado-May, 2013a, 2015b). El énfasis está en procesos cognitivos que conducen a la creación, innovación y transferencia de conocimiento.

El enfoque en fortalecer los procesos cognitivos propios de la cultura, la identidad, la autoestima, la superación académica y la calidad en su formación implica bastante trabajo y esfuerzo de los estudiantes. Esta tendencia contrasta fuertemente con la posición de los políticos, que creen en el concepto de la «deuda histórica» con los indígenas, lo cual se ha convertido en un mensaje consistente e insistente, de tal forma que se ha creado una política paternalista que mucho ha perjudicado la herencia cultural y cognitiva de los indígenas. La política paternalista se expresa en acciones como la de no procurar una educación de alto nivel, no tener profesores de alto nivel, no hacer evaluaciones adecuadas para conocer el nivel de aprendizaje, no motivar a la superación, facilitar los rezagos y la fácil obtención de un título universitario. En contraste con el paternalismo, los integrantes de la comunidad universitaria llevan a cabo acciones diseñadas para ayudar con efectos positivos, como describen Mullainathan y Shafir (2013); por ejemplo, los estudiantes ayudan a sus pares que necesitan encontrar dónde vivir, cómo adaptarse en JMM, cómo integrarse a la vida universitaria y cómo enfrentar los trámites, gestiones, para las becas, seguro médico, viajes de práctica, trámites de cambio de programa educativo, etc.

El énfasis que la UIMQRoo puso en los procesos cognitivos también se explica por la necesidad de hacer investigaciones académicas. Por un lado, existe la percepción en las IES no interculturales de que la investigación en las UI no tiene peso científico sólido ni es competitivo; por otro, si la investigación que se hace en las UI no tiene una identidad diferente de la que se hace en las IES convencionales, no solo se refuerza la percepción antes descrita, sino que se favorece a quienes argumentan que las UI no tienen razón de existir. Por tanto, es necesario enfocar esfuerzos para desarrollar conceptos y metodologías de investigación intercultural. No es totalmente nuevo: existen ejemplos de procesos de construcción de conocimiento interculturales. La agroecología es un buen ejemplo (Rosado-May, 2015b). En las artes y humanidades hay muchos ejemplos de resultados interculturales que no han recibido reconocimiento ni se conocen los procesos que condujeron a esos resultados. La sinergia entre diferentes formas 
de construcción de conocimiento que ofrece el modelo intercultural tiene un enorme potencial que México debe aprovechar para entender y atender los grandes retos que tiene, no solo con los indígenas sino con toda la sociedad.

Desde el punto de vista estructural, son varios los retos que aún persisten en las UI de México. Uno de ellos es que los planes y programas de gobierno federal y estatal tienen mucha prisa para obtener resultados cuantitativos (e.g. número de estudiantes matriculados) y afectan el paso natural, seguro y fuerte, en la consolidación de un modelo educativo nuevo y prometedor. Otro reto a superar es el de la formación y experiencia de los directivos, profesores e integrantes de los órganos colegiados; debe prevalecer la contratación de excelentes perfiles, con potencial para desarrollar el modelo en la dirección correcta, y eliminar las malas prácticas de gestión universitaria (Rosado-May 2016, pp. 66-78), así como eliminar la discriminación y falta de respeto a los derechos humanos y aprovechar los saberes acumulados por los sabios locales. Las personas que integran los órganos colegiados normalmente no reciben capacitación, esta es una práctica que debe mejorar. Aunado a la formación y capacitación intercultural, es importante impulsar cuerpos académicos y programas de posgrado en materia de educación intercultural. Sería un gran retroceso si la maestría en educación intercultural en la UIMQRoo, única en México, no se fortalece ni se suma a esfuerzos internacionales. Pero no solo educación, sino otras áreas de conocimiento demandan formación de posgrado, con el enfoque intercultural, tanto en las ciencias exactas, naturales, sociales, económico-financieras, políticas y humanidades. El país debe promover el desarrollo de posgrado en estos campos del conocimiento; no hacerlo implica altos costos de oportunidad tanto financiera como social.

\section{REFERENCIAS BIBLIOGRÁFICAS}

Abercrombie, Nicholas, Stephen Hill y Bryan S. Turner (2014). The Penguin Dictionary of Sociology, 4th edition. Oxford: Oxford University Press.

Aguiló Bonet y J. Antoni (2009). La universidad y la globalización alternativa: justicia cognitiva, diversidad epistémica y democracia de saberes. Nómadas. Revista Crítica de Ciencias Sociales y Jurídicas, 22 (2009.2). Publicación electrónica de la Universidad Complutense. Recuperado de http://pendientedemigracion.ucm. es/info/nomadas/22/antoniaguilo.pdf

Balcázar, Fabricio E., Luciano Berardi y Tina Taylor-Ritzler (2011). El «privilegio de los blancos»: otra fuente de dominación social de las clases privilegiadas. Espacios en Blanco, Serie Indagaciones, 21(1), 85-110. 
Bertely Busquets, María (2011). Educación superior intercultural en México. Perfiles Educativos, XXXIII, 66-77. Recuperado de http://www.redalyc.org/ pdf/132/13221258007.pdf

BID (2012). Implementación de la estrategia para el desarrollo indígena. Lecciones de la revisión de portafolio. Nueva York: Banco Interamericano de Desarrollo. Recuperado de https://publications.iadb.org/bitstream/handle/11319/5533/Spanish.pdf? sequence $=9$

Caballero Rodríguez, Katia (2013). La formación del profesorado universitario y su influencia en el desarrollo de la actividad profesional. Revista de Docencia Universitaria 11(2), 391-412.

Carrillo Trueba, César (2009). El racismo en México. México, Conaculta, Colección Tercer Milenio.

Casillas Muñoz, Lourdes y Laura Santini Villar (2006). Universidad intercultural. Modelo educativo. Serie Documentos. México: Secretaría de Educación Pública, Coordinación General de Educación Intercultural y Bilingüe.

Castellanos Guerrero, Alicia (2000). Antropología y racismo en México. Desacatos, (4), 53-79.

CDI (2017). Indicadores de la población indígena. Comisión para el Desarrollo de los Pueblos Indígenas, Gobierno de México. Recuperado el 12 de marzo de 2017 de www.gob.mx/cdi/documentos/indicadores-de-la-poblacion-indigena

CGEIB (2017). Matrícula de estudiantes en universidades interculturales de México. Coordinación General de Educación Intercultural y Bilingüe, Secretaría de Educación Pública. Recuperado el 10 de marzo de 2017 de http://eib.sep.gob.mx/ diversidad/universidades-interculturales

Chavajay, Pablo y Barbara Rogoff (2002). Schooling and traditional collaborative social organization of probem solving by Mayan mothers and children. Developmental Psychology, 38, 55-66. https://doi.org/10.1037/0012-1649.38.1.55

Chávez Galindo, Ana María y Héctor Hiram Hernández Bringas (2003). ¿Cuál es el tamaño de la población indígena en México? Revista de la Universidad de México, noviembre, 91-95.

CONAPO (2001). La población de México en el nuevo siglo. Tamaño de la población indígena mexicana. México, Consejo Nacional de Población. Recuperado el 12 de marzo de 2017 de http://www.conapo.gob.mx/es/CONAPO/La_poblacion_ de_Mexico_en_el_nuevo_siglo

Crosby, Faye J. (2004). Affirmative action is dead; long live affirmative action. Current Perspectives in Psychology. New Haven y Londres: Yale University Press. 
Gaskins, Suzanne (1999). Children's daily lives in a Mayan Village: A case study of culturally constructed roles and activities. En A. Göncü (ed.), Children's engagement in the world (pp. 25-61). Cambridge: Cambridge University Press.

Gaskins, Suzanne (2003). From corn to cash: Change and continuity within Mayan families. Ethos, 31(2): 248-273. https://doi.org/10.1525/eth.2003.31.2.248

Gaskins, Suzanne y Ruth Paradise (2010). Learning through observation in daily life. En D.F. Lancy, J. Bock y S. Gaskins (eds.), The anthropology of learning in childhood (pp. 100-140). Lanham, MD: Alta Mira Press.

González González, Floriberto, Francisco J. Rosado-May y Gunther Dietz (coords.) (2017). La gestión de la educación superior intercultural en México. Retos y perspectivas de las universidades interculturales. Iguala, Guerrero: Universidad Autónoma de Guerrero y El Colegio de Guerrero A.C. y Editorial Trinchera.

IFAD (2003). Indigenous peoples and sustainable development. Discussion paper. Roma, Italia: International Fund for Agricultural Development. Recuperado el 10 de marzo de 2017 de https://www.ifad.org/documents/10180/cff46e5c-19a64077-b1ea-94a83888bfbf

INEGI (2000). Censo nacional de población y vivienda. México, Instituto Nacional de Estadística y Geografía. Recuperado el 13 de marzo de 2017 de http://www3. inegi.org.mx/sistemas/biblioteca/ficha.aspx?upc=702825006519

INEGI (2015) Encuesta intercensal 2015. México: Instituto Nacional de Estadística y Geografía. Recuperado el 13 de marzo de 2017 de http://www.inegi.org.mx/est/ contenidos/proyectos/accesomicrodatos/encuestas/hogares/especiales/ei2015/

López, José y John Scott (2000). Social structure. Buckingham y Philadelphia: Open University Press.

Mallea, John R. y Jonathan C. Young (1984). Cultural diversity and Canadian education. Ottawa: Carleton University Press.

Mato, Daniel (2012). Educación superior y pueblos indígenas y afrodescendientes en América Latina. Constituciones, leyes, políticas públicas y prácticas institucionales. En Daniel Mato (coord.), Educación superior y pueblos indígenas y afrodescendientes en América Latina. Normas, políticas y prácticas (pp. 13-98). Caracas: Instituto Internacional de la Unesco para la Educación Superior en América Latina y el Caribe (IESALC-Unesco).

Mejía Pérez, Gustavo (2016). Las universidades públicas estatales de apoyo solidario: entre el aislamiento y la precariedad. Nexos, noviembre 30. Recuperado el 13 de marzo de 2017 de http://educacion.nexos.com.mx/?p=384

Mendoza-Rojas, Javier (2015). Ampliación de la oferta de educación superior en México y creación de instituciones públicas en el periodo 2001-2012. Revisa 
Iberoamericana de Educación Superior, VI (16), 3-32. https://doi.org/10.1016/j. rides.2015.04.001

Morales y Gómez, Juan M. (2009). Historia del estudio de la administración pública en México. México: Universidad Autónoma del Estado de México, Miguel Ángel Porrúa y Cámara de Diputados.

Moreno, Patricia, Margarita Campos y Guillermo Rodríguez (2016). La configuración de una comunidad de investigadores educativos en una universidad pública mexicana: de la individualidad a la colaboración. Formación Universitaria, 9(5), 65-74. https://doi.org/10.1016/j.rides.2015.04.001

Mullainathan, Sendhil y Eldar Shafir (2013). Scarcity. Nueva York: Picador y Henry Holt and Co.

Paradise, Ruth (1987). Learning trough social interaction: The experience and development of the Mazahua self in the context of the market. Doctoral dissertation, University of Pennsylvania.

Paradise, Ruth (1994). The autonomous behavior of indigenous students in classroom activities. En A. Álvarez y P. del Río (eds.), Education as cultural construction (pp. 89-95). Madrid: Fundación Infancia y Aprendizaje.

Paradise, Ruth y Marie De Haan (2009). Responsibility and reciprocity: Social organization of Mazahua learning practices. Anthropology y Education Quarterly, 40(2), 187-204. https://doi.org/10.1111/j.1548-1492.2009.01035.x

PNUD (2010). Informe sobre el desarrollo humano de los pueblos indígenas de México. México: Programa de Naciones Unidas para el Desarrollo. Recuperado el 12 de marzo de 2017 de http://planipolis.iiep.unesco.org/upload/Mexico/Mexico_ HDR 2010.pdf

Rogoff, Barbara (1981). Schooling and the development of cognitive skills. En H.C. Triandis y A. Heron (eds.), Handbook of cross-cultural psychology, 4, 233-294. Rockleigh, NJ: Allyn y Bacon.

Rogoff, Barbara (1990). Apprenticeship in thinking: Cognitive development in social context. Nueva York: Oxford University Press.

Rogoff, Barbara (2003). The cultural nature of human development. Nueva York: Oxford University Press.

Rogoff, Barbara (2014). Learning by observing and pitching in to family and community endeavors: An orientation. Human Development, 57(2-3), 69-81. https://doi. org/10.1159/000356757

Rogoff, Barbara, Benosh Najafi y Rebeca Mejía-Arauz (2014). Constellations of cultural practices across generations: Indigenous American heritage and learning by observing and pitching in. Human Development, 57(2-3), 82-95. https://doi. org/10.1159/000356761 
Rosado-May, Francisco J, Martha V. Kú Martínez y Nelsy R. Cituk Poot (2016). Hacia la transformación social como resultado de la formación de los egresados de la Universidad Intercultural Maya de Quintana Roo. Un enfoque etnográfico. IV Congreso Mexicano de Antropología Social y Etnología, Santiago de Querétaro, Querétaro, México; 13 de octubre.

Rosado-May, Francisco J. (2012). Una perspectiva intercultural al concepto de tutoría académica. El caso de la UIMQRoo. En I. Deance y V. Vázquez Valdés, Aulas diversas. Experiencias sobre educación intercultural en América (pp. 65-90). Quito: ABYA/YALA Universidad Politécnica Salesiana, Deance-Vázquez y Universidad Intercultural Maya de Quintana Roo.

Rosado-May, Francisco J. (2013a). Indigenous education. Which way to go? The intercultural model for higher education developed in Mexico. II International Meeting Canada-Mexico on Indigenous Education, University of Lethbridge, Canada, June 22nd.

Rosado-May, Francisco J. (2013b). Experiencias y visión de futuro de la Universidad Intercultural Maya de Quintana Roo. Aportaciones del modelo intercultural a la sociedad. En A. Wind (coord.), Experiencias y visiones para el futuro de las universidades indígenas en el mundo (pp.157-172). La Paz, Bolivia: Instituto Internacional de Integración-Convenio Andrés Bello.

Rosado-May, Francisco J. (2015a). Papel de la visión/percepción en la gobernanza de una universidad intercultural: impacto en la calidad, indicadores y el desarrollo institucional. II Congreso Internacional de Educación Intercultural, Universidad Intercultural Maya de Quintana Roo, José María Morelos, Quintana Roo, México, 12-14 de agosto.

Rosado-May, Francisco J. (2015b). The intercultural origin of agroecology: contributions from México. En V. Ernesto Méndez, Christopher M. Bacon, Roseann Cohen y Stephen R. Gliessman (eds.), Agroecology, a transdisciplinary, participatory and action oriented approach. Boca Raton: CRC Press. https://doi. org/10.1201/b19500-9

Rosado-May, Francisco J. (2016). Educación superior sostenible en Quintana Roo, México. Elementos para la creación de un sistema articulado con la investigación, crecimiento económico y desarrollo. Chetumal, Quintana Roo, México: Malú Villarreral Ediciones.

Rosado-May, Francisco J. (2017). Los retos y oportunidades de guiar inteligencia con inteligencia. El modelo de educación superior intercultural en Quintana Roo. En Floriberto González González, Francisco J. Rosado-May y Gunther Dietz (coords.), La gestión de la educación superior intercultural en México. Retos y perspectivas de las universidades interculturales. Iguala, Guerrero, México: Universidad Autónoma de Guerrero, El Colegio de Guerrero A.C. y Ediciones Trinchera. 
Rosado-May, Francisco J. y Marí C. Osorio Vázquez (2014). Construyendo interculturalidad a través de movilidad académica estudiantil: experiencias UIMQRoo. En S. Dydou Aupetit (coord.), Los programas de educación superior indígena en América Latina y en México. Caracas, Venezuela: Unesco-Instituto Internacional para la Educación Superior en América Latina y el Caribe.

Rubio Oca, Julio (coord.) (2006). La política educativa y la educación superior en México, 1995-2006: un balance. México D.F.: Fondo de Cultura Económica y Secretaría de Educación Pública.

Schmelkes, Sylvia (2013). Educación y pueblos indígenas: problemas de medición. Revista Internacional de Estadística y Geografía, enero-abril, 4(1).Recuperado el 9 de marzo de 2017 de http://www.inegi.org.mx/RDE/RDE_08/RDE_08_ Art1.html

SEP (2006). Programa de mejoramiento del profesorado PROMEP: un primer análisis de su operación e impacto en el proceso de fortalecimiento académico de las universidades públicas. México: Secretaría de Educación Pública.

Shaw, Rajib, Yukiko Takeuchi, Noralene Uy and Anshu Sharma (2009). Indigenous knowledge, disaster risk reduction. Policy note. Bangkok,Thailand; Kyoto, Japan; Puram New a, India: European Union, International Strategy for Disaster Reduction, Kyoto University y Seeds. Recuperado el el 25 de febrero de 2017 de http://www.unisdr.org/files/8853_IKPolicyNote.pdf

Sobrevila, Claudia (2008). The role of indigenous peoples in biodiversity conservation. The natural but often forgotten partners. Washington DC: The World Bank.

Toledo, Víctor (2013). Indigenous people and biodiversity. En Encyclopedia of Biodiversity (S.A. Levin, ed.). 2da ed. Ámsterdan: Elsevier Inc. https://doi.org/10.1016/ B978-0-12-384719-5.00299-9

UIMQRoo (2010). Reglamento interior de la Universidad Intercultural Maya de Quintana Roo. Capítulo II, Del Consejo Directivo. Recuperado el 30 de septiembre de 2017 de http://www.uimqroo.edu.mx/Documentos/NuestraUniversidad/reglamentoInterior.pdf

Velazco Cruz, Saúl y Bruno Baronnet (2016). Racismo y escuela en México: reconociendo la tragedia para intentar la salida. Diálogos sobre Educación, 7(13), 1-17.

WEF (2017). Global risk report, 2017. 12 ed. Ginebra: World Economic Forum.

Wilson, Shawn (2008). Research is ceremony: Indigenous research methods. Halifax: Fernwood Publishing.

Zogaib Achcar, Elena (2000). El programa de mejoramiento del profesorado (Promep) y sus críticas. Revista Mexicana de Ciencias Politicas y Sociales, XLIV(178), $135-157$. 